\title{
Extending item response theory to online homework
}

\author{
Gerd Kortemeyer* \\ Lyman Briggs College and Department of Physics and Astronomy, Michigan State University,
} East Lansing, Michigan 48824, USA

(Received 6 January 2014; published 27 May 2014)

\begin{abstract}
Item response theory (IRT) becomes an increasingly important tool when analyzing "big data" gathered from online educational venues. However, the mechanism was originally developed in traditional exam settings, and several of its assumptions are infringed upon when deployed in the online realm. For a largeenrollment physics course for scientists and engineers, the study compares outcomes from IRT analyses of exam and homework data, and then proceeds to investigate the effects of each confounding factor introduced in the online realm. It is found that IRT yields the correct trends for learner ability and meaningful item parameters, yet overall agreement with exam data is moderate. It is also found that learner ability and item discrimination is robust over a wide range with respect to model assumptions and introduced noise. Item difficulty is also robust, but over a narrower range.
\end{abstract}

DOI: 10.1103/PhysRevSTPER.10.010118

\section{INTRODUCTION}

Item response theory (IRT) offers the opportunity for data-driven development and evaluation of assessment items, such as homework, practice, concept inventory, and exam problems. The technique is increasingly used in physics education research, for example, to examine the validity of concept tests $[1,2]$, and also to evaluate online homework problems (see Ref. [3]).

As opposed to classical test theory, IRT assumes that learners have a latent trait of "ability" beyond their overt score on a particular set of test items. One learner's inherent constant ability may result in different scores on different sets of test items, depending on how difficult, well written, meaningful, or representative these items are. Trivially, even high-ability students are likely going to fail on an overly difficult item. More disturbingly, high-ability students are less likely than low-ability students to succeed on ill-written items that have subtle distractions, missing assumptions, or undesired complications that lower-ability students may not even detect. However, to model the ability of learners, IRT still depends on a particular learner's performance on an item to actually reflect that particular learner's ability to correctly solve it. Thus, for IRT to be reliable, there are a number of implicit assumptions, which are usually fulfilled in standard exam settings.

- Learners receive no correctness feedback while taking the exam, and thus effectively have only one attempt to get a problem correct.

\footnotetext{
*kortemey@msu.edu

Published by the American Physical Society under the terms of the Creative Commons Attribution 3.0 License. Further distribution of this work must maintain attribution to the author $(s)$ and the published article's title, journal citation, and DOI.
}

PACS numbers: 01.50.H-, 01.40.G-, 01.40.-d, 01.50.Kw

- The setting is high stakes, so learners will refrain from guessing if at all possible.

- Learners receive no outside help.

- The setting is controlled, so copying of solutions is minimized.

- The identity of the learner is verified, so the person taking the exam is indeed who they claim to be.

- The assessment is summative and designed to evaluate the ability of the learner.

Under these "experimental conditions," the resulting clean signal is "a learner with ability $\theta$ (on some scale) has a probability $p_{i}(\theta)$ to get item $i$ correct." All of these assumptions are likely violated in online homework situations, where noise is introduced by the following scenarios.

- Most systems offer correctness feedback and allow for multiple attempts.

- Some learners are guessing answers even if minimal effort could result in correct solutions [4].

- At times, learners receive outside help from online forums, teaching assistants, older students, etc., some of which is productive and some of which is not $[5,6]$.

- Some students are copying answers [7], most likely from students who already successfully solved the problem.

- Occasionally, students are solving each other's homework problems.

- The assessment is formative and designed to increase the ability of the learner.

Scenarios related to copying and guessing result in the learner's score on an item not reflecting that particular learner's own best-effort ability. Design features like correctness feedback and multiple attempts support the goal of mastery-based learning, but from a pure 
measurement perspective make scores less meaningful: even low-ability students might eventually get the items correct. And finally, the nature of formative assessment and associated learning could (or should) make ability a "moving target" that improves over the course of the semester-the question essentially is "nature versus nurture" of latent physics ability, where the constant ability model of IRT implies "nature."

It is thus not clear that IRT can be expanded into the online homework realm. The question is of increasing importance as larger and larger online courses are viewed as sources of "big data," and analytics is seen as an essential component of the next generation publication of educational materials. How large is the error introduced by each of the confounding factors? In the online realm, is IRT still a meaningful measure of problem difficulty and discrimination?

But IRT is not only relevant for material development and evaluation. In large courses, IRT has the potential of early detection of students at risk. Ideally, low-ability learners should be identified before summative assessment venues, so that there is still a chance for remediation and additional support. Thus, data from online homework could be the key to helping these learners-but how reliable is IRT based on online formative assessment data for the determination of learner ability?

Section II presents the population and setting of the study, as well as the employed statistical techniques. Section III illustrates the problem by comparing an IRT analysis of the exam data with an analysis of homework data. Section IV investigates the effect of having multiple attempts in the online realm, while Sec. V investigates guessing and copying behavior. Finally, Sec. VI provides the conclusions of this study.

\section{DATA AND METHOD}

\section{A. The course}

To investigate the influence of violating some of the basic assumptions of IRT, this study considers a largeenrollment (256 student) physics course for scientists and engineers. This course recently increased the number of exams throughout the semester (12 exams and one final) [8], which resulted in 184 exam items. The course also has 13 online homework assignments with a total of 401 problems, which are administered via LON-CAPA [9].

Like many other assessment systems, LON-CAPA randomizes problems-students get different versions of the same problem, e.g., different numbers, graphs, formulas, scenarios, or images [9]. Problems can be rendered differently for different settings, e.g., a problem that was written as a free-response numerical problem appears as a multiple choice problem on printed exams, where either the system or the author specify randomized wrong answer choices.

Both exams and homework had a mixture of conceptual and numerical questions. Figure 1 shows an example of a homework problem from the course, while Fig. 2 shows an example of an exam problem. Exams were administered in a lecture hall using bubble sheets and multiple choice questions, where each student had a different randomized version of the problems in order to minimize copying. Also, for homework each student had a different version of the problems to at least avoid blind copying of answers. LON-CAPA was configured to give immediate correctness feedback and to allow for multiple attempts (in the case of multiple-choice questions, usually two or three attempts, in the case of free-response numerical questions, 99 attempts, i.e., essentially unlimited). No grade penalty was given for using multiple attempts.

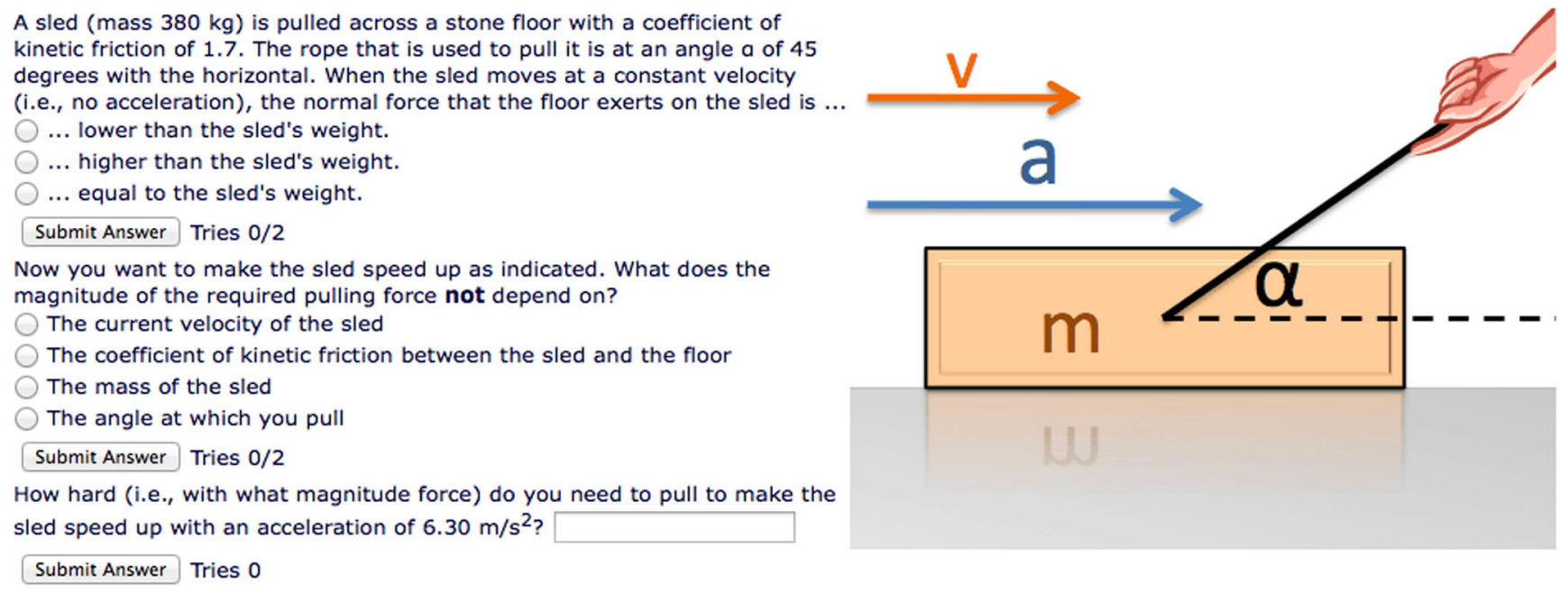

FIG. 1. An example of a homework problem from the course under investigation. Different students have different masses and angles, as well as different multiple-choice options in different order. In this course, students have 99 tries on the free-form part of the problem. Students can use any physical unit with the right dimension. 
Two particles of different mass, $m_{1}>m_{2}$, collide totally inelastically. They initially have identical speeds, however, the direction is at an angle $\theta$ above and below the $\mathrm{x}$-axis, respectively, as shown. Which one of the indicated final velocities is correct?

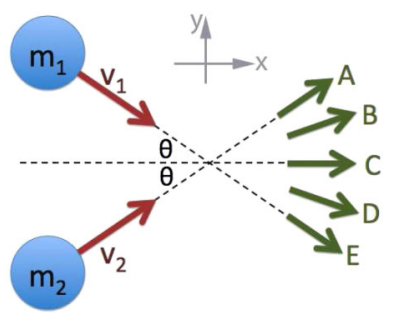

$1 p t$

1.A $\bigcirc \mathrm{A}$, along the initial velocity of particle 2 .

$\mathrm{B} \bigcirc \mathrm{B}$, between the direction of the initial velocity of particle 2 and the $\mathrm{x}$-axis.

$\mathrm{C} \bigcirc \mathrm{C}$, along the $\mathrm{x}$-axis

$\mathrm{D} \bigcirc \mathrm{D}$, between the $\mathrm{x}$-axis and the direction of the initial velocity of particle 1 .

$\mathbf{E} \bigcirc \mathrm{E}$, along the initial velocity of particle 1.

$1 p t$ If the initial speeds are $v_{1}=v_{2}=8.4 \mathrm{~m} / \mathrm{s}$ and the angle $\theta$ is 41 degrees, what is the $\mathrm{x}$-component of the final velocity? (in $\mathrm{m} / \mathrm{s}$ )

$\begin{array}{lllll}\text { 2. } & \mathbf{A} \bigcirc 3.2 & \mathbf{B} \bigcirc 4.1 & \mathbf{C} \bigcirc 5.1 & \mathbf{D} \bigcirc 6.3 \\ \mathbf{E} \bigcirc 7.9 & \mathbf{F} \bigcirc 9.9 & \mathbf{G} \bigcirc 12 & \mathbf{H} \bigcirc 15\end{array}$

FIG. 2. An example of an exam problem from the course under investigation. Different students have different relative masses $\left(m_{1}<m_{2}\right.$ versus $\left.m_{1}>m_{2}\right)$, as well as different speeds and angles $\theta$. The numerical answer options and predetermined physical unit are the automated exam rendering of a free-form problem.

\section{B. IRT model}

IRT models the probability $p_{i}(\theta)$ for a learner with a certain latent ability $\theta$ to successfully solve item $i$. As a function of ability, one expects the graph of $p(\theta)$ for good items to exhibit a stretched $S$ shape: low-ability students have a low chance of getting the problem correct, while high-ability students have a high chance. Different IRT models vary in how this so-called "item characteristic curve" is parametrized, most notably how many parameters are used for fitting the data. For example, a one parameter logistic model (1PL), also known as the Rasch model, uses the logistic function

$$
p_{i}(\theta)=\frac{\exp \left(\theta-b_{i}\right)}{1+\exp \left(\theta-b_{i}\right)}
$$

to model an item. Here, the parameter $b_{i}$ models the difficulty of the item, as it moves the turning point between most likely getting the item correct versus incorrect toward higher ability $\theta$.

Unless noted otherwise, this study is using the widely accepted two parameter logistic (2PL) model [10], which introduces an additional discrimination parameter $a_{i}$ :

$$
p_{i}(\theta)=\frac{\exp \left[a_{i}\left(\theta-b_{i}\right)\right]}{1+\exp \left[a_{i}\left(\theta-b_{i}\right)\right]} .
$$

The parameter $a_{i}$ essentially controls the slope of the function, i.e., how rapidly the chance of getting the item correct increases with increasing ability.

Determining the individual learner abilities $\theta_{j}$ and the item parameters $a_{i}$ and $b_{i}$ is an iterative process of minimizing the discrepancy between the predictions $P_{i}\left(\theta_{j}\right)$ and the actual performance of learner $j$ on item $i$; i.e., it is an optimization process of maximizing the likelihood of the ability estimate. Beginning with some starting values, the vectors $\theta_{j}, a_{i}$, and $b_{i}$ hopefully converge to achieve this maximum likelihood estimate; however, convergence is not guaranteed. Calculations are performed using the latent trait model (LTM) package [11] within the R statistical software system [12].

\section{THE PROBLEM}

\section{A. Item characteristics}

Figure 3 illustrates the difference in IRT results between controlled exam settings (IRT's natural habitat, left-hand panel) and online homework settings (right-hand panel), Each curve represents one problem and shows the likelihood of getting the problem correct as a function of the ability of the student. Both measures of difficulty and discrimination can be extracted from these typical item characteristic curves: the farther to the left the curve goes from low to high probability, the easier the problem, and the steeper the curve, the more strongly the item discriminates between low- and high-ability learners.

The exam outcome is rather typical: there are obviously some better and worse problems (most notably one problem that only half of the best students in the class are likely going to solve), but overall, the vast majority of the items exhibit the typical stretched $S$ shape. The homework outcome, on the other hand, is widely distributed. In particular, the high density of curves in the upper left quadrant of the figure shows that even low-ability students get many problems correct.

It would be very easy to attribute this simply to the multiple allowed attempts, and in fact, IRT studies of online homework tended to attempt to compensate by considering only the first attempt (see Ref. [13]), i.e., considering the problem correct only if it was solved on the first try. However, as Fig. 4 shows, using this approach, the curves spread out farther, making the result even less meaningful.

While exam and homework problems are similar in character (except that all exam questions are multiple choice), there is little direct overlap, as the exact same problem rarely appears on both homework and exams. Thus, unfortunately, in order to move beyond the qualitative comparisons of Figs. 3 and 4, a paired comparison between the IRT parameters is not possible. 

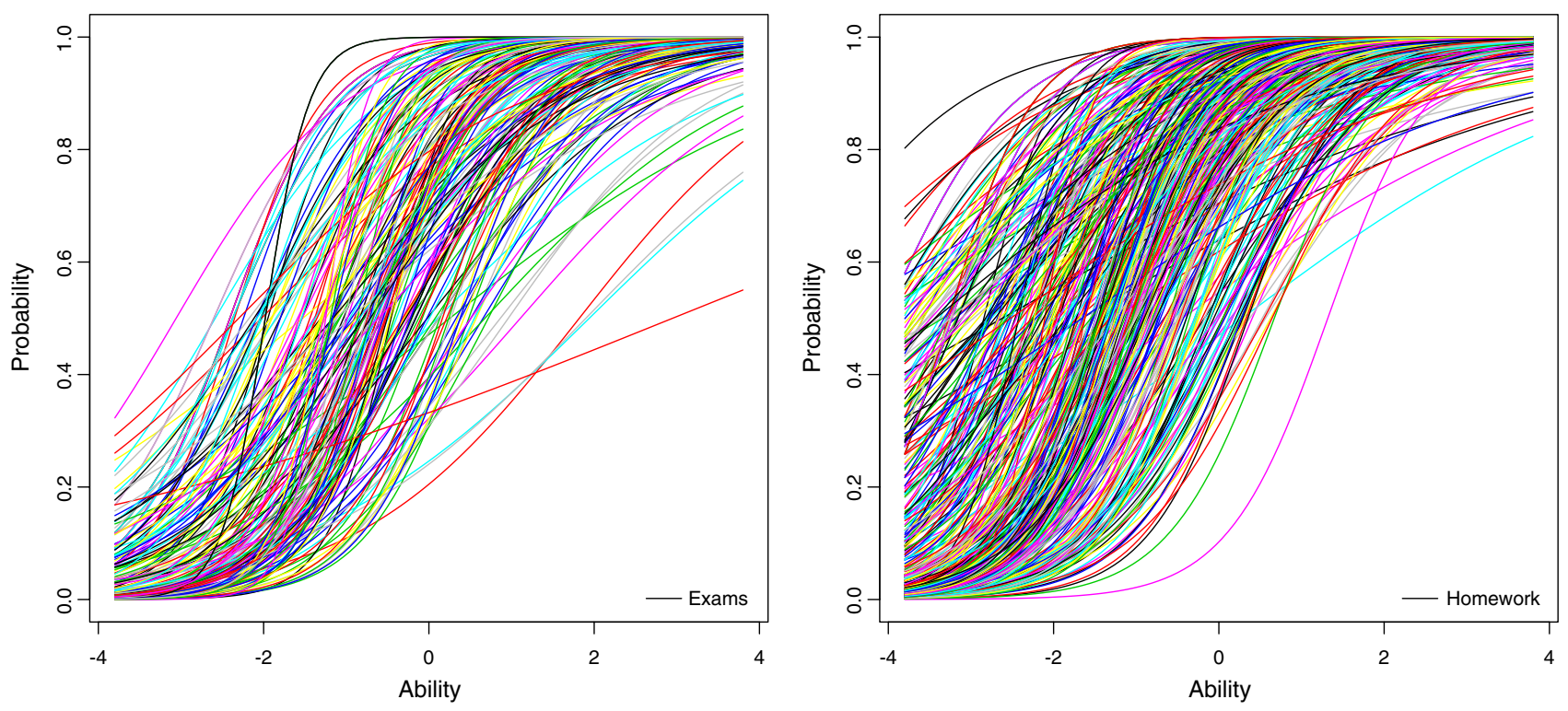

FIG. 3. Item characteristic curves for exam items (left) and homework items (right). Homework items were considered correct if the learner solved them eventually, regardless of number of attempts.

\section{B. Learner ability}

Without considering the larger question of whether or not multiple-choice exams are a valid measure of learning, for the purposes of this study, the learner ability estimates based on the exam data will be considered a reliable measure. After all, these exams will largely determine the grade in the course, and the closer the estimates obtained from homework agree with the exam-based estimates, the more useful they are for early detection of students at risk.

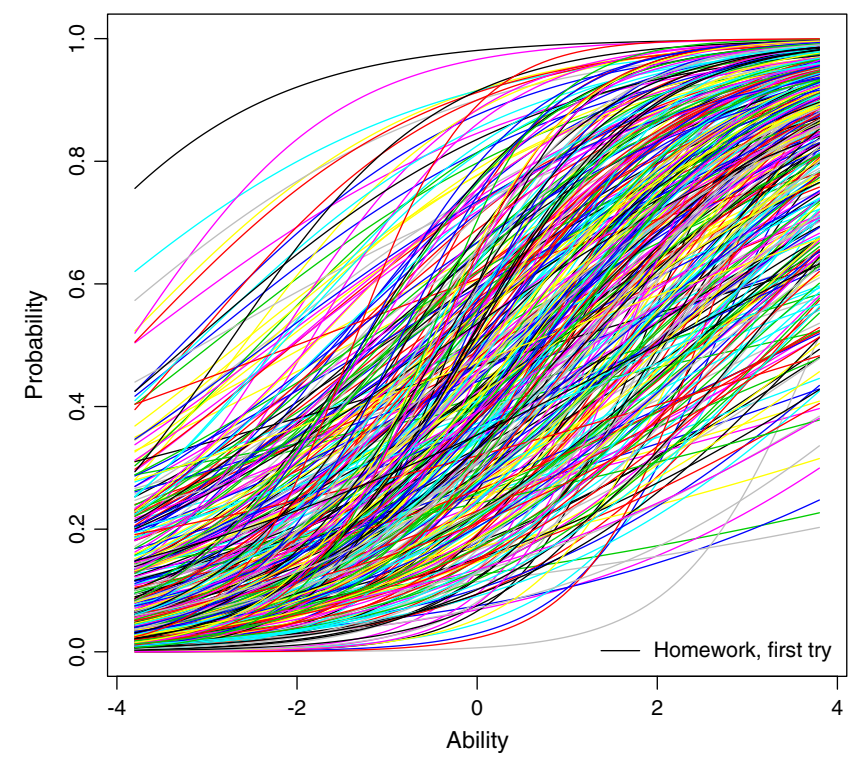

FIG. 4. Item characteristic curves for homework. Homework items were considered correct if they were solved on the first attempt.
The data allow for pairwise comparison of ability score estimates, as the same students did work on homework and exams. With $j$ being the student, and with $\theta_{E, j}$ and $\theta_{H, j}$ being the ability estimates of the student for exams and homework, respectively, a measure for the overall similarity of the estimates is the cosine of the angle $\alpha$ between the "ability vectors"; i.e.,

$$
\cos \alpha=\frac{\vec{\theta}_{E} \vec{\theta}_{H}}{\left|\vec{\theta}_{E}\right|\left|\vec{\theta}_{H}\right|} .
$$

A value of one would indicate perfect agreement, a value of zero would mean the outcomes are "perpendicular" (meaning, nonrelated), and a value of negative one would mean the exact opposite relationship. The correspondence turns out to be not very large: it is 0.64 between exams and homework considering all attempts, and 0.67 when considering only the first attempt.

\section{INFLUENCE OF MULTIPLE ATTEMPTS}

\section{A. Thresholds for number of attempts}

In order to better understand the influence of imposing a threshold $N$ for the maximum number of considered attempts, different thresholds were employed. In other words, a problem was considered correct only if the number of attempts that the student needed to solve the problem was smaller than or equal to the respective threshold $N$. As it turns out, the $2 \mathrm{PL}$ fit did not converge for several maximum numbers of attempts (namely, $N=4$, $8,9,10,11,12$, and 13) and was unstable for others (namely, $N=3,6$, and 7). This can be due to some items 


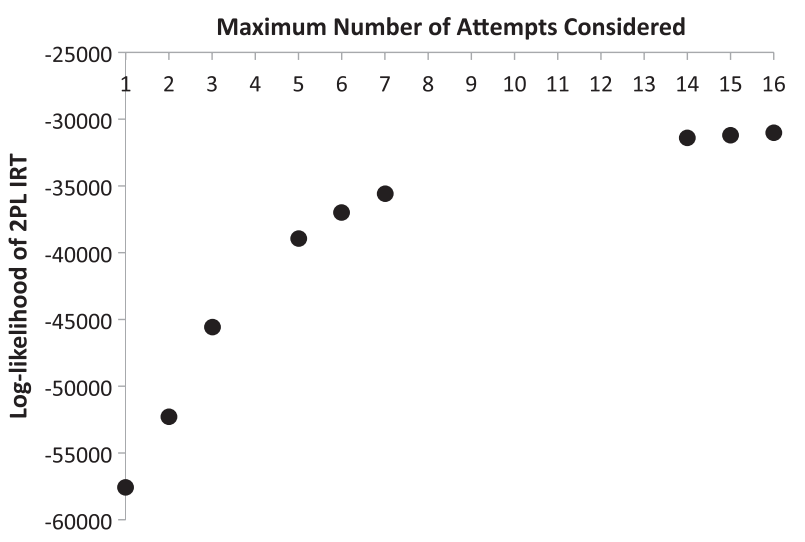

FIG. 5. Log-likelihood of the 2PL IRT analysis of the homework data as a function of the maximum number $N$ of considered attempts. Missing data points indicate that the fit did not converge. For comparison, without imposing a threshold on the number of attempts, the log-likelihood is -30070 .

having extreme properties, which lead to diverging discrimination parameters. The problem is usually solved by removing these "dysfunctional items" from consideration and restarting the optimization process. However, in the interest of comparability, the item pool was left unchanged.

\section{Log-likelihood}

IRT studies usually report the natural logarithm of the achieved maximum likelihood of the ability estimate, which is going to be a negative number: the smaller the magnitude, the higher the maximum likelihood. This loglikelihood can roughly be understood as a measure of the quality of the fit. Figure 5 shows this value as a function of the imposed threshold. This value increases (i.e., the fit becomes more reliable) the more attempts are considered, and it eventually approaches the log-likelihood of the threshold-free analysis, i.e., -30070 (for comparison, the log-likelihood of the exam data 2PL fit is -20930).

\section{Item difficulty}

Figure 6 shows the average difficulty of the items as a function of the maximum number $N$ of considered attempts. The 2PL fits yield an error estimate of the item difficulties, and the error bars in the figure indicate the average of these error estimates. As expected, the problems get "easier" the more attempts are considered (the exception, $N=7$, is one of the unstable solutions).

\section{Item discrimination}

Figure 7 shows the average discrimination of the items as a function of the maximum number of considered attempts, once again the error bars indicating the average of the error estimates. The result is interesting: one may have expected the discrimination of the items would go down the more attempts are considered. In other words, a

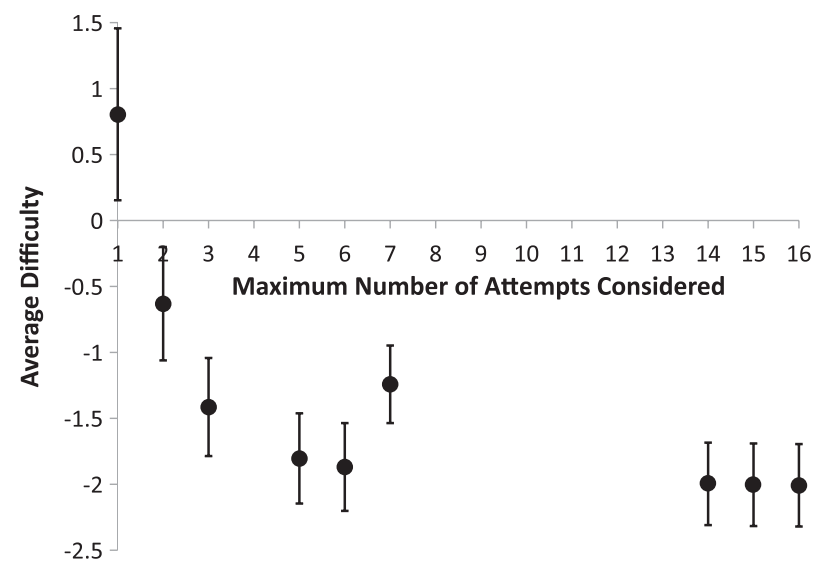

FIG. 6. Average difficulty of the homework items as a function of the maximum number $N$ of considered attempts. Missing data points indicate that the fit did not converge. The error bars indicate the average of the standard errors on the difficulties.

simple assumption would be that low- and high-ability students would best get separated by looking at the first attempt only ("good students would get the problem correct on the first try"), or maybe the second try (as even good students might be sloppy in their initial attempt). However, this is clearly not the case, which underlines the formative assessment character of online homework. If students indeed were expected to get everything right on the first attempt, there would be no need to assign the problem. It is clear that the discrimination actually increases as more and more attempts are considered, which may suggest that good students are distinguished by their tenacity to keep working on a homework problem rather than their immediate genius.

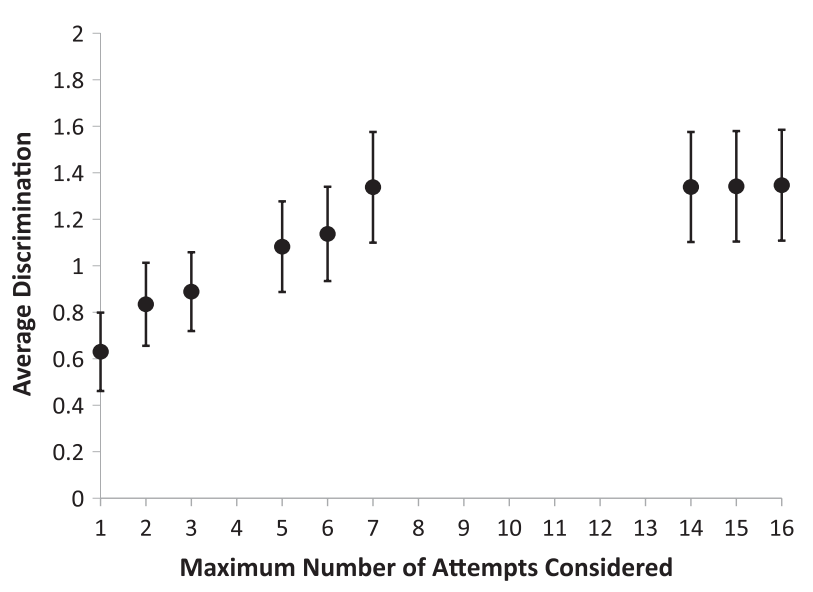

FIG. 7. Average discrimination of the homework items as a function of the maximum number $N$ of considered attempts. Missing data points indicate that the fit did not converge. The error bars indicate the average of the standard errors on the discriminations. 


\section{Learner ability}

The cosine similarity between the ability estimates of the exam and homework data is essentially independent of the number of considered attempts, it varies between 0.67 for $N=1$ and 0.66 for $N=16$. Thus, the choice of $N$ has almost no influence on the quality of the estimate of the learner ability. For comparison, when considering all attempts, the cosine similarity was 0.64 (see Sec. III B), and thus not much additional information is gained by limiting the number of considered tries.

\section{B. Partial credit for multiple attempts}

Another approach is to allocate different amounts of partial credit for a solved problem, depending on how many attempts were needed to solve it. In this case, IRT needs to be extended to accept item results other than just zero and one, and one such model is the generalized partial credit model [14], which is also implemented in the LTM package. The model deals only with polytomous data, so continuous mappings of numbers of attempts $n$ to partial credit $p$ [e.g., $p=1 / n$ or $p=\exp (1-n)]$ are not supported. To explore this mechanism, a simple mapping of $p=6$ for having solved the problem on the first try, $p=5$ for the second try, etc., to $p=2$ for successful completion after any number of tries was chosen. For technical reasons, $p=1$ was used for unsuccessful attempts, as the package translates $p=0$ into "not answered."

The resulting fit had a low log-likelihood (-126370). Item discriminations were reported on a different scale than in the dichotomous model, but the cosine similarity is very high: it is 0.94 for considering either all attempts or up to $N=16$ attempts (see Sec. IV A 3), and 0.95 for considering only the first attempt $(N=1)$; regarding the item discrimination, the dichotomous and polytomous models are thus almost equivalent. Regarding learner abilities, the fit yielded a cosine similarity of 0.66 with the exam data, which is almost exactly equivalent to considering all attempts or the findings in Sec. IVA 4-once again, both models are almost equivalent.

For our data (256 students, 401 items, and 6 partial credit bins), computation time increased by almost 2 orders of magnitude compared to the simple dichotomous model without gaining additional information about the items or the learners. Thus, the additional computational complexity does not appear to be justified compared to simply considering the dichotomous model of whether or not the learner eventually solved the problem.

\section{Discussion of multiple attempts}

Essentially all attempts of remedying the effect of offering multiple attempts by limiting the number of considered attempts or employing some partial credit scheme were nonor even counterproductive. Considering only the first attempt did not lead to any improvements, which is likely due to the typical "guessing" behavior of students, which leads to essentially wasting the first attempt half of the time [4] (and 25\%-35\% on later tries [8]) — the students know that they have multiple attempts and act accordingly. Beyond considering only the first attempt, the agreement with exam data regarding problem discrimination and learner ability is equivalent for practical considerations, and essentially nothing is gained beyond simply looking at the dichotomous model of whether or not the students eventually solved the problem.

The good news is that this robustness of results proves the value of using IRT in the online homework context, since the results of simple descriptive statistics would vastly depend on these choices: for example, as more and more attempts are considered, the classical descriptive values of the abilities of the learners increase-a nonsensical situation that would demand somewhat arbitrary choices of thresholds or partial credit schemes.

\section{GUESSING AND COPYING}

\section{A. Three parameter model}

When estimating the effect of guessing, a natural first approach is to employ a three parameter model (3PL), where the third parameter $c_{i}$ models the guessing behavior [10]:

$$
p_{i}(\theta)=c_{i}+\left(1-c_{i}\right) \frac{\exp \left[a_{i}\left(\theta-b_{i}\right)\right]}{1+\exp \left[a_{i}\left(\theta-b_{i}\right)\right]} .
$$

Essentially, $c_{i}$ gives even low-ability students a chance of getting the item correct.

As it turns out, though, this third parameter leads to only minimal improvement of the fit, regardless of whether only the first or all attempts are considered. When considering only the first attempt, some of the problem discrimination gets lost in favor of the guessing parameter, but this effect almost completely vanishes when considering all attempts. In both cases, the average guessing parameter is small (0.031 when considering only the first attempt, and 0.019 when considering all attempts). The cosine similarity of the learner ability with the exam results once again remains largely unchanged.

Initially, particularly when considering only the first attempt, this outcome seems counterintuitive-do students not guess after all? However, the guessing modeled by the three parameter model is nonrandom guessing in the case of difficult problems. This is not what students are doing: their first attempt is frequently truly random or at most reflecting some "gut feeling," regardless of the actual difficulty of the problem [4]. In addition, IRT expects a limited number of answer choices, where the hit-and-miss ratio is determined by the number of bubbles, not a free response with essentially infinitely many answer choices.

However, preliminary experiments with a nonstandard 3PL of the form 


$$
p_{i}(\theta)=\left(1-c_{i}\right) \frac{\exp \left[a_{i}\left(\theta-b_{i}\right)\right]}{1+\exp \left[a_{i}\left(\theta-b_{i}\right)\right]}
$$

in an attempt of modeling losing (instead of gaining) points due to guessing also failed to improve the fit.

In summary, a three parameter model with a guessing parameter is hardly useful in the online homework scenario and in fact might be overfitting the data.

\section{B. Earlier parts of the semester}

Students often begin a new course with good intentions and high scores in the diligence cluster of epistemological surveys, only to have these good intentions fall by the wayside as the semester progresses [15]. It is thus reasonable to assume that guessing and copying behavior increases over the course of the semester, which was indeed found to be the case [7].

To investigate if a signature of this can be found in the homework data, only the first quarter of the semester was considered. As it turns out, the cosine similarity of the learner abilities to the exam data decreases when considering all attempts (0.60), but it increases when considering only the first attempt (0.72). In other words, in the first weeks of the semester, the first attempt on a homework problem is still meaningful-in fact, the extracted cosine similarity of the learner ability to the exam data is the highest found in this study. As the semester progresses, it appears that considering all attempts increases the quality of the learner ability estimates (to 0.64, see Sec. IV A 4), while considering only the first attempt becomes less useful (the cosine similarity decreases to 0.67 ). These effects are minimal, and the absolute agreement is moderate at best in any case, but they do support the emerging picture of the effect of copying and guessing.

The good news is that an early-warning system for learners at risk might indeed be possible. Using IRT, already a few weeks into the semester, meaningful learner ability estimates can be obtained (in this case, considering only the first attempt does improve the quality of the estimate).

\section{Modeling guessing and copying}

While the effect of offering multiple attempts can to some degree be "undone" in the homework data by either limiting the number of considered attempts (Sec. IVA) or employing a partial credit model (Sec. IV B), guessing and copying cannot be subtracted from the homework data beyond limiting the analysis to the initial weeks of the course where the students still "behaved better" (Sec. V B). Instead, to assess their effect, they can be artificially added to the exam data; assuming that the exam data are "clean," guessing and copying behavior can be simulated by manipulating the student-to-item response matrix.

In an exam scenario, copying would mean copying somebody else's answers without necessarily knowing if these answers are right or wrong. The behavior would need to be modeled by increasingly copying data from one row of the student-to-item matrix into another row; i.e., increased copying would make one student's item response row increasingly similar to the row of another student. Copying of online homework with immediate feedback is different: a student would not copy somebody else's incorrect answer. Thus, in the online homework scenario, guessing and copying are quite similar, but the success rate is much higher for the latter.

Thus, both guessing and copying of online homework with immediate correctness feedback can be modeled approximately with the same two parameter manipulation of the "clean" exam data. The two parameters are percentage of occurrence, i.e., what percent of the problems the student is guessing on or copying, and success rate, i.e., how often this behavior yields a correct answer. The success rate ranges from low percentages for "stabs in the dark" and somewhat higher percentages for "educated guesses" to very high percentages for surefire solutions, i.e., solutions that have been copied or adapted from somebody else's correct solution (note that the problems are randomized, so the success rate for copying is likely not $100 \%$ ).

Using a $10 \%$ success rate for educated guesses, a $90 \%$ success rate for copying randomizing homework, and a $50 \%$ success rate for comparison, the decay of the "clean" estimates from the original exam data was traced for different occurrence rates between $0 \%$ and $100 \%$. Figure 8 shows the effect on the problem difficulty estimates. If more than a third of the problem solutions are guessed or copied, the cosine similarity of the difficulty estimates rapidly decreases. In other words, problem difficulties are very sensitive to the introduced noise.

On the other hand, Figs. 9 and 10 show good agreement with the original discrimination and ability estimates until more then two-thirds of the problem solutions are guessed or copied. Going back to the results from the real

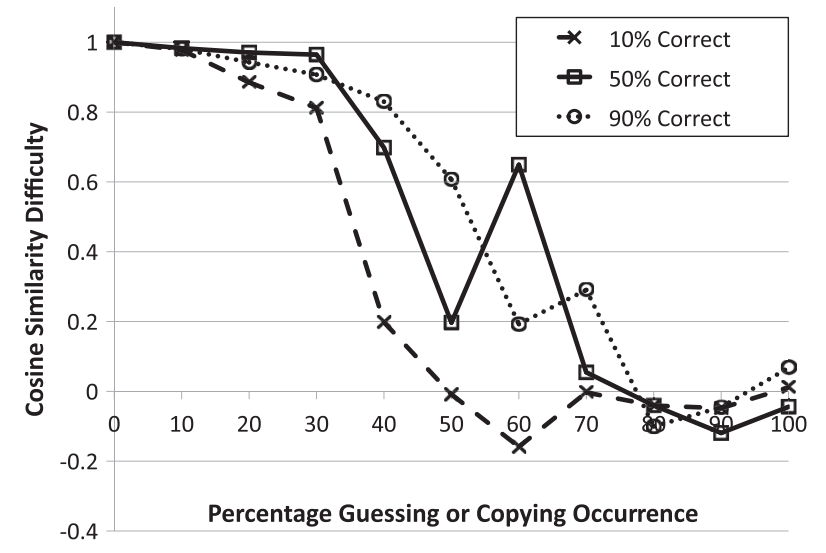

FIG. 8. Cosine similarity of the item difficulties from the "clean" exam values as a function of simulated copy or guess occurrence. Values are shown for a success rate of $10 \%$ (“educated guessing"), 50\%, and 90\% (“copying"). 


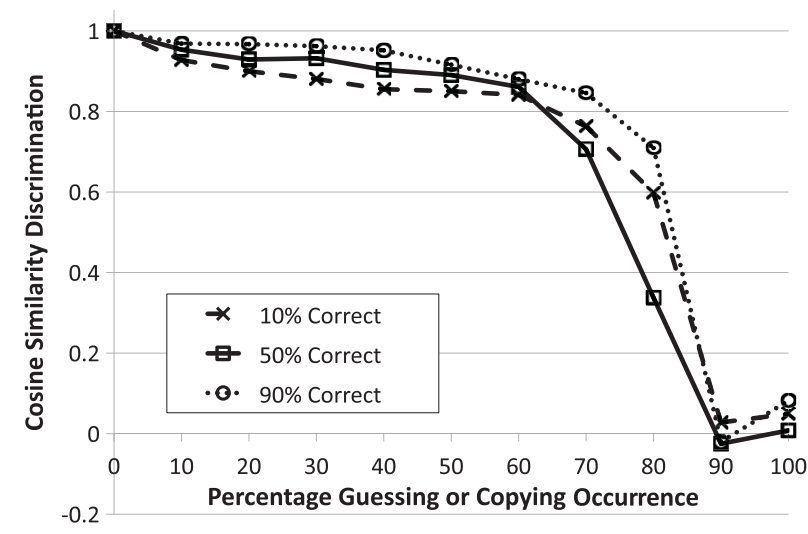

FIG. 9. Cosine similarity of the item discriminations from the "clean" exam values as a function of simulated copy or guess occurrence. Values are shown for a success rate of $10 \%$ ("educated guessing"), 50\%, and 90\% ("copying").

homework data, where cosine agreements of learner ability with exam data are in the range of 0.60 to 0.72 , one might be tempted to conclude that guessing and copying are more prevalent than assumed thus far-however, that is not taking into account that the homework and exam problems are different, as well as the other confounding effects of multiple attempts and free-response solutions.

Once again, the good news is the robustness of IRT estimates in the presence of noise. While the difficulty estimates might break down somewhat early, the discrimination and learner ability estimates show remarkable reliability. Data on actual copying and guessing percentages for comparison purposes are naturally difficult to obtain. In a study carried out in an another semester of this same course, students reported that they consulted "cheating sites" for $10 \%-20 \%$ of the problems [8], and it is likely that $50 \%$ of the initial and $25 \%-35 \%$ of the subsequent homework attempts are guesses [4,8]. Palazzo et al. [7]

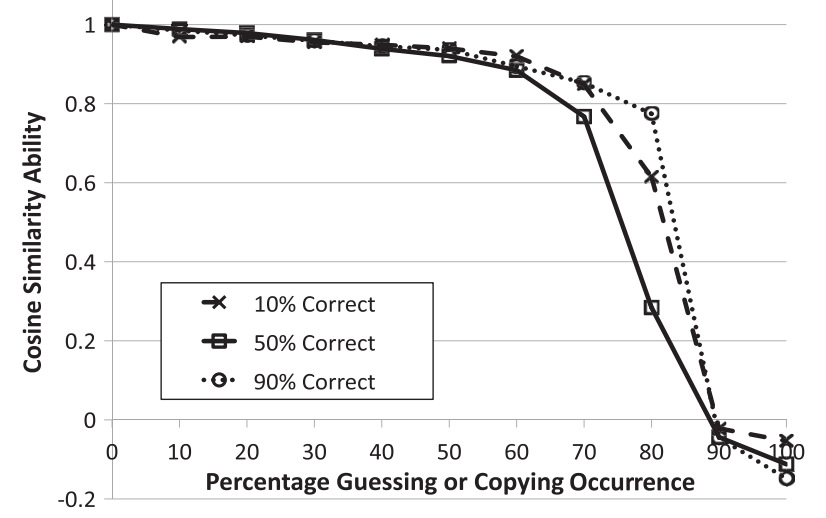

FIG. 10. Cosine similarity of the learner abilities from the "clean" exam values as a function of simulated copy or guess occurrence. Values are shown for a success rate of $10 \%$ ("educated guessing"), 50\%, and 90\% (“copying"). report similar 10\%-20\% average copying percentages for courses at MIT, however, with wide variations between individual students and depending on problem position in assignments, time of semester, and proximity of deadlines. Based on these copying and guessing percentages, given the findings from this simple model, even difficulty estimates are still reasonably reliable. Only the 50\% guessing percentage on first attempts [4] yields unacceptable reliability of problem difficulties, which once again underlines the questionable benefit of considering only the first attempt in IRT calculations.

A more sophisticated model should take into account the differentiated findings of Palazzo et al. [7] instead of just considering average percentages. In particular, it should take into account that some students guess or copy and others do not (instead of the indiscriminate guessing or copying in the current model), and that maybe low-ability students are more prone to guess or that they would likely copy from high-ability students. The main result from this simple model is that IRT parameters and ability estimates are relatively robust with respect to the noise introduced by copying and guessing over varying ranges that fortunately encompass estimates of actual student behavior in typical courses, but then very rapidly break down-there is no graceful degradation.

\section{CONCLUSIONS}

Extending IRT to online homework scenarios poses considerable challenges. It should not simply be assumed that the model transfers, and this study attempted to quantify the effects of violating the original assumptions of the model.

When using IRT to estimate learner ability, results obtained from online homework show the correct trends compared to exam data, but the absolute agreement is moderate. Within these limitations, however, IRT is remarkably robust with respect to online homework offering multiple attempts, and stable in terms of noise introduced from copying and guessing up to a point. When it comes to item parameters, discrimination is less affected by the homework environment than difficulty; however, when IRT breaks down, it does so rapidly.

The agreement of homework and exam data, as well as the quality of item parameters, is not improved by using more complicated models or filtering of the data. In particular, trying to model guessing or introducing partial credit does not yield additional information. The effect of considering only the first attempt on homework wears out over the course of the semester. Thus, overall it seems that simple is better: using a straightforward two parameter logistic model on the full homework (only considering if the problems were solved eventually) appears to be about as fruitful as the introduction of additional assumptions or constraints. 
[1] L. Ding and R. Beichner, Approaches to data analysis of multiple-choice questions, Phys. Rev. ST Phys. Educ. Res. 5, 020103 (2009).

[2] C. N. Cardamone, J. E. Abbott, S. Rayyan, D. T. Seaton, A. Pawl, and D. E. Pritchard, Item response theory analysis of the mechanics baseline test, AIP Conf. Proc. 1413, 135 (2012).

[3] Y.-J. Lee, D. J. Palazzo, R. Warnakulasooriya, and D. E. Pritchard, Measuring student learning with item response theory, Phys. Rev. ST Phys. Educ. Res. 4, 010102 (2008).

[4] G. Kortemeyer, Gender differences in the use of an online homework system in an introductory physics course, Phys. Rev. ST Phys. Educ. Res. 5, 010107 (2009).

[5] G. Kortemeyer, An analysis of asynchronous online homework discussions in introductory physics courses, Am. J. Phys. 74, 526 (2006).

[6] G. Kortemeyer, Correlations between student discussion behavior, attitudes, and learning, Phys. Rev. ST Phys. Educ. Res. 3, 010101 (2007).

[7] D. J. Palazzo, Y.-J. Lee, R. Warnakulasooriya, and D. E. Pritchard, Patterns, correlates, and reduction of homework copying, Phys. Rev. ST Phys. Educ. Res. 6, 010104 (2010).

[8] J. T. Laverty, W. Bauer, G. Kortemeyer, and G. Westfall, Want to reduce guessing and cheating while making students happier? Give more exams!, Phys. Teach. 50, 540 (2012).

[9] G. Kortemeyer, E. Kashy, W. Benenson, and W. Bauer, Experiences using the open-source learning content management and assessment system LON-CAPA in introductory physics courses, Am. J. Phys. 76, 438 (2008).

[10] A. Birnbaum, in Statistical Theories of Mental Test Scores, edited by F. M. Lord and M. R. Novick (Addison-Wesley, Reading, Mass, 1968), pp. 374-472.

[11] D. Rizopoulos, LTM: An R package for latent variable modelling and item response theory analyses, J. Stat. Software 17, 1 (2006).

[12] R Development Core Team, R: A Language and Environment for Statistical Computing (R Foundation for Statistical Computing, Vienna, 2008), ISBN 3-900051-07-0.

[13] Y. Bergner, S. Dröschler, G. Kortemeyer, S. Rayyan, D. Seaton, and D. Pritchard, in Proceedings of the International Educational Data Mining Society (IEDMS), Chania, Greece, 2012, Vol. 5, pp. 95-102, http:// educationaldatamining.org/EDM2012/uploads/procs/Full_ Papers/edm2012_full_4.pdf.

[14] E. Muraki, A generalized partial credit model: Application of an EM algorithm, Appl. Psychol. Meas. 16, 159 (1992).

[15] E. F. Redish, R. N. Steinberg, and J. M. Saul, Student expectations in introductory physics, Am. J. Phys. 66, 212 (1998). 